\title{
Cross sections for gluon + gluon $\rightarrow$ heavy quarkonium + gluon
}

\section{Citation}

Gastmans, R., W. Troost, and Tai Tsun Wu. 1987. "Cross Sections for Gluon + Gluon

$\rightarrow$ Heavy Quarkonium + Gluon." Physics Letters B 184 (2-3): 257-60. https:// doi.org/10.1016/0370-2693(87)90578-8.

\section{Permanent link}

http://nrs.harvard.edu/urn-3:HUL.InstRepos:41555797

\section{Terms of Use}

This article was downloaded from Harvard University's DASH repository, and is made available under the terms and conditions applicable to Other Posted Material, as set forth at http:// nrs.harvard.edu/urn-3:HUL.InstRepos:dash.current.terms-of-use\#LAA

\section{Share Your Story}

The Harvard community has made this article openly available.

Please share how this access benefits you. Submit a story.

Accessibility 


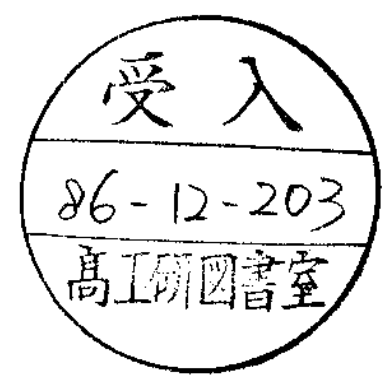

CERN-TH. $4586 / 86$

CROSS-SECTIONS FOR GLUON + GLUON $\rightarrow$ HEAVY QUARKONIUM + GLUON

\author{
R. Gastmans ${ }^{*}$ ) and W. Troost ${ }^{+}$) \\ Institute for Theoretical Physics \\ University of Le uven, B-3030 Le uven \\ and \\ Tai. Tsun Wu") \\ CERN - Geneva \\ and \\ Gordon McKay Laboratory \\ Harvard University, Cambridge MA 92138
}

\title{
A B S T R A C T
}

Using the helicity amplitude formalism, we derive simple cross-section formulae for the production of heavy quarkonia through the process $g+g \rightarrow\left(2 S+L_{L_{J}}\right)+g$ when $L=S$ or $P$. Within the framework of perturbative $\mathrm{CD}$, these subprocesses are important for the hadroproduction of heavy quarkonia.

*) Onderzoeksleider N.F.W.o., Belgium

+) Bevoegdverklaard Navorser N.F.W.O., Belgium.

") Work supported in part by the United States Department of Energy under grant DE-FG02-84ER40158.

CERN-TH. $4586 / 86$

November 1986 
Hadroproduction of the $J / \psi$ has recently been observed at the CERN $\bar{p} \bar{p}$ collider [1]. It is to be expected that the hadroproduction of heavy quarkonia, in general, will be an important source of information on various aspects of high-energy physics: it allows one to study $B-\bar{B}$ mixing $[1-3]$, to search for extra b quarks [4], and to test perturbative QCD [5].

Within the framework of perturbative QCD, the production of heavy quarkonia is described by several subprocesses, such as $g g \rightarrow{ }^{2 S+1} \mathrm{~L}, \mathrm{~J} \rightarrow{ }^{2 \mathrm{~S}+1_{\mathrm{L}}}$, $\mathrm{g} \mathrm{q} \rightarrow{ }^{2 S+1} \mathrm{~L}_{\mathrm{J}} \mathrm{q}, \mathrm{g} \overline{\mathrm{q}} \rightarrow{ }^{2 S+1} \mathrm{~L}_{\mathrm{J}} \overline{\mathrm{q}}, \mathrm{q} \overline{\mathrm{q}} \rightarrow{ }^{2 S+1} \mathrm{~L}_{\mathrm{J}} \mathrm{g}, \mathrm{gg} \rightarrow{ }^{2 S+L_{L_{J}}} \mathrm{~g}$, etc. These processes are easily evaluated using the standard Feynman diagram techniques [5], except for the case $g \mathrm{~g} \rightarrow{ }^{2 S+1} L_{J} g$, described by the Feynman diagrams of Fig. 1. In this case, the number of Feynman diagrams is rather large and the covariant summation over the polarization degrees of freedom leads to long and cumbersome expressions which, for the ${ }^{3} \mathrm{p}$ channels, were handled by symbolic manipulation programs .

In this letter, we present our cross section formulae for the process

$$
g\left(k_{1}\right)+g\left(k_{2}\right) \rightarrow{ }^{2 S+1} L_{J}+g\left(k_{3}\right)
$$

where $k_{1}, k_{2}$ and $k_{3}$ represent the momenta of the gluons. Our results are limited to the lowest order, i.e., $\alpha_{s}^{3}$, not including the much more complicated higher-order QCD corrections. The formulae were obtained using the helicity formalism [6] developed earlier, where one introduces an explicit representation of the three gluon polarization vectors. E.g.,

$$
\begin{aligned}
& \not_{1}^{ \pm}=N\left[k_{1} k_{2} k_{3}\left(1 \mp \gamma_{5}\right)+k_{3} k_{2} k_{1}\left(1 \pm \gamma_{5}\right)\right], \\
& N=(2 s t u)^{-1 / 2},
\end{aligned}
$$

where $s=\left(k_{1}+k_{2}\right)^{2}, t=\left(k_{2}-k_{3}\right)^{2}$, and $u=\left(k_{1}-k_{3}\right)^{2}$. 
$-2-$

In this way, we obtain simple expressions for the two independent helicity amplitudes of $g . g \rightarrow g q \vec{q}$. With the method of Guberina, Kühn, Peccei, and Rück1 [7], it then becomes a straightforward task to obtain the cross section formulae for process (1). Further details of this procedure will be presented in a forthcoming paper.

We simply list our results:

$$
\begin{aligned}
& { }^{1} S_{0}: \frac{d \sigma}{d t}=\frac{\pi \alpha_{s}^{3} R_{0}^{2}}{M s^{2}} \frac{P^{2}\left(M^{8}-2 M^{4} P+P^{2}+2 M^{2} Q\right)}{Q\left(Q-M^{2} P\right)^{2}}, \\
& { }^{3} S_{1}: \frac{d \sigma}{d t}=\frac{10 \pi \alpha_{s}^{3} R_{0}^{2}}{9 s^{2}} \frac{M\left(P^{2}-M^{2} Q\right)}{\left(Q-M^{2} P\right)^{2}}, \\
& { }_{1} \mathcal{P}_{1}: \frac{d \sigma}{d t}=\frac{40 \pi \alpha_{s}^{3} R_{1}^{\prime 2}}{3 M s^{2}} \frac{-M^{10} P+M^{6} P^{2}+Q\left(S M^{8}-7 M^{4} P+2 P^{2}\right)+4 M^{2} Q^{2}}{\left(Q-M^{2} P\right)^{3}}, \\
& 3 P_{0}: \frac{d \sigma}{d t}=\frac{4 \pi \alpha_{s}^{3} R_{1}^{\prime 2}}{M^{3} s^{2}} \frac{1}{Q\left(Q-M^{2} \mathcal{P}\right)^{4}}\left[9 M^{4} \mathcal{P}^{4}\left(M^{8}-2 M^{4} \mathcal{P}+\mathcal{P}^{2}\right)\right. \\
& -6 M^{2} P^{3} Q\left(2 M^{8}-5 M^{4} P+P^{2}\right)-P^{2} Q^{2}\left(M^{8}+2 M^{4} P-P^{2}\right) \\
& \left.+2 M^{2} P Q^{3}\left(M^{4}-P\right)+6 M^{4} Q^{4}\right] \\
& { }^{3} P_{1}: \frac{d \sigma}{d t}=\frac{12 \pi \alpha_{s}^{3} R_{1}{ }^{2}}{M^{3} s^{2}} \frac{P^{2}\left[M^{2} P^{2}\left(M^{4}-4 P\right)-2 Q\left(M^{8}-5 M^{4} P-P^{2}\right)-15 M^{2} Q^{2}\right]}{\left(Q-M^{2} P\right)^{4}} \\
& { }^{3} P_{2}: \frac{d \sigma}{d t}=\frac{4 \pi \alpha_{s}^{3} R_{1}^{\prime 2}}{M^{3} s^{2}} \frac{1}{Q\left(Q-M^{2} P\right)^{4}}\left[12 M^{4} P^{4}\left(M^{8}-2 M^{4} \mathcal{P}+P^{2}\right)\right. \\
& -3 M^{2} P^{3} Q\left(8 M^{8}-M^{4} P+4 P^{2}\right)+2 P^{2} Q^{2}\left(-7 M^{8}+43 M^{4} P+P^{2}\right) \\
& \left.+M^{2} P Q^{3}\left(16 M^{4}-61 P\right)+12 M^{4} Q^{4}\right] \text {, }
\end{aligned}
$$


with $P=s t+t u+u s, q=s t u$, and $M^{2}=s+t+u$, i.e., the (mass) ${ }^{2}$ of the produced resonance. In these formulae, $R_{0}$ is related to the ( $\left.q \vec{q}\right)$ wave function at the origin and is given by

$$
R_{0}^{2}=M^{2} r\left({ }^{3} S_{1} \rightarrow e^{+} e^{-}\right) / 4 \alpha^{2} e_{q}^{2}
$$

while $R_{1}^{\prime}$ is related to its derivative at the origin. Using the quarkonium potential of ref.[8] with $\Lambda=0.2 \mathrm{GeV}$, one finds for the (ci) system

$$
R_{1}^{\prime 2} / M_{x}^{2}=0.006 \cdot(6 e V)^{3}
$$

where ${ }^{M} \chi^{\text {is the mass of a }}{ }^{3} p$ state.

The cross section formulae (3) can be written in many different ways. An alternative set of formulae is:

${ }^{1} S_{0}: \frac{d \sigma}{d t}=\frac{n \alpha_{s}^{3} R_{0}^{2}}{8 M s^{2}}\left[\frac{M^{4}-s^{2}-t^{2}-u^{2}}{\left(s-M^{2}\right)\left(t-M^{2}\right)\left(u-M^{2}\right)}\right]^{2} \cdot \frac{M^{8}+s^{4}+t^{4}+u^{4}}{s t u}$,

${ }^{3} S_{1}: \frac{d \sigma}{d t}=\frac{5 \pi \alpha_{s}^{3} R_{0}^{2} M}{9 s^{2}}\left[\frac{s^{2}}{\left(t+M^{2}\right)^{2}\left(u-M^{2}\right)^{2}}+\frac{t^{2}}{\left(u-\mu^{2}\right)^{2}\left(s-M^{2}\right)^{2}}+\frac{u^{2}}{\left(s-M^{2}{ }^{2}\left(t-M^{2}\right)^{2}\right.}\right]$,

${ }^{1} \mathcal{P}_{1}: \frac{d \sigma}{d t}=\frac{20 \pi \alpha_{s}^{3} R_{1}^{\prime 2}}{3 M s^{2}} \frac{1}{\left[\left(s-M^{2}\right)\left(t-M^{2}\right)\left(u-M^{2}\right)\right]^{2}}$

$$
\left\{M^{4}\left(s^{2}+t^{2}+u^{2}+M^{4}\right)+\frac{2 s t u\left[s^{4}+t^{4}+u^{4}+M^{4}\left(s^{2}+t^{2}+u^{2}\right)+2 M^{8}\right]}{\left(s-M^{2}\right)\left(t-M^{2}\right)\left(u-M^{2}\right)}\right\},
$$


$-4-$

$$
\begin{aligned}
3 P_{0}: \frac{d \sigma}{d t}= & \frac{2 n \alpha_{s}^{3} R_{1}^{\prime 2}}{m^{3} s^{2}} \frac{1}{\left[\left(s-M^{2}\right)\left(t-m^{2}\right)\left(u-m^{2}\right)\right]^{2}} \\
\{ & 8 M^{2}\left[\frac{t u\left(t^{4}-t^{2} u^{2}+u^{4}\right)}{\left(s-m^{2}\right)^{2}}+\frac{u s\left(u^{4}-u^{2} s^{2}+s^{4}\right)}{\left(t-m^{2}\right)^{2}}+\frac{s t\left(s^{4}-s^{2} t^{2}+t^{4}\right)}{\left(u-m^{2}\right)^{2}}\right] \\
& +4 M^{4}\left[M^{2}(s t+t u+u s)-5 s t u\right] \\
& +(s t+t u+u s)^{2}\left[\frac{g M^{8}}{s t u}+\frac{1}{\left(s-m^{2}\right)\left(t-m^{2}\right)\left(u-m^{2}\right)}\left(s M^{4}\left(s^{2}+t^{2}+u^{2}\right)\right.\right. \\
& \left.\left.\left.-16 m^{2} s t u+\left(1-9 M^{2}\left(\frac{1}{s}+\frac{1}{t}+\frac{1}{u}\right)\right)\left(s^{4}+t^{4}+u^{4}\right)\right)\right]\right\},
\end{aligned}
$$

$$
\begin{aligned}
{ }^{3} P_{1}: \frac{d \sigma}{d t}= & \frac{12 \pi \alpha_{s}^{3} R_{1}^{\prime 2}}{M^{3} s^{2}} \frac{1}{\left[\left(s-\mu^{2}\right)\left(t-M^{2}\right)\left(u-\mu^{2}\right)\right]^{2}} \\
& \left\{M^{2}\left[\frac{t^{2} u^{2}\left(t^{2}+u^{2}\right)}{\left(s-M^{2}\right)^{2}}+\frac{u^{2} s^{2}\left(u^{2}+s^{2}\right)}{\left(t-M^{2}\right)^{2}}+\frac{s^{2} t^{2}\left(s^{2}+t^{2}\right)}{\left(u-M^{2}\right)^{2}}\right]\right. \\
& \left.+\frac{2\left(s^{2} t^{2}+t^{2} u^{2}+u^{2} s^{2}\right)\left(s^{2} t^{2}+t^{2} u^{2}+u^{2} s^{2}+M^{2} s t u\right)}{\left(s-M^{2}\right)\left(t-M^{2}\right)\left(u-M^{2}\right)}\right\},
\end{aligned}
$$

$$
\begin{aligned}
3 P_{2}: \frac{d \sigma}{d t}= & \frac{4 \pi \alpha_{s}^{3} R_{1}^{\prime 2}}{M^{3} s^{2}} \frac{1}{\left[\left(s-M^{2}\right)\left(t-M^{2}\right)\left(u-M^{2}\right)\right]^{2}} \\
& \left\{M^{2}\left[\frac{t^{2} u^{2}\left(t^{2}+4 t u+u^{2}\right)}{\left(s-m^{2}\right)^{2}}+\frac{u^{2} s^{2}\left(u^{2}+4 u s+s^{2}\right)}{\left(t-m^{2}\right)^{2}}+\frac{s^{2} t^{2}\left(s^{2}+4 s t+t^{2}\right)}{\left(u-m^{2}\right)^{2}}\right]\right. \\
& +12 M^{2}\left[3\left(s^{3} t+t^{3} u+u^{3} s+s t^{3}+t u^{3}+u s^{3}\right)+4 m^{2} s t u\right] \\
& +\frac{2\left(s t+t u+u s-M^{4}\right)(s t+t u+m s)^{2}}{\left(s-M^{2}\right)\left(t-M^{2}\right)\left(u-M^{2}\right)}[s t+t u+u s \\
& \left.\left.-24 M^{4}-6 M^{2}\left(s t+t u+m s-M^{4}\right)\left(\frac{1}{s}+\frac{1}{t}+\frac{1}{u}\right)\right]\right\} .
\end{aligned}
$$


It is seen that the helicity formalism [6] is capable of yielding simple and useful formulae for the process $g \mathrm{~g} \rightarrow{ }^{2 \mathrm{~S}+1_{\mathrm{L}}} \mathrm{g}$. Such formulae are expected to have many applications. An especially outstanding example is related to the very recent study of Glover, Halzen and Martin [9] on the physics from $J / \psi$-tags in $\mathrm{p} \overline{\mathrm{p}}$ collisions. The present formulae can lead to a significant saving of computer time, perhaps by a factor of 5 , to obtain the cross section for $g g \rightarrow \chi g$, offering the possibility of more refined studies.

\section{ACKNOWLEDGEMENTS}

We are very grateful to Professor Alfred Mueller and to Professor Francis Halzen for drawing our attention to the need for simple formulae for these processes. Also, many useful conversations with Professor Francis Halzen are gratefully acknowledged. One of us (TTW) wishes to thank Professor John Ellis, Professor Maurice Jacob and many other members of the Theory Division for their kind hospitality at CERN . 


\section{REFERENCES}

[1] UAI Collaboration, T. Markiewicz, in Proceedings of the XXIII International High Energy Conference, Berkeley, July 1986.

[2] J. Ellis, M. K. Gaillard, D. V. Nanopoulos and S. Rudaz, Nucl. Phys. B131 (1977) 285 .

[3] UAl Collaboration, G. Arnison et al., Phys. Lett. 155B (1985) 442.

[4] E. W. N. Glover, F. Halzen and A. D. Martin, Phys. Lett. 176B (1986) 480.

[5] Chang Chao-Hsi., Nucl. Phys. B172 (1980) 425;

R. Baier and R. Rück1, Phys. Lett. Bl02 (1981) 364; Nucl. Phys. B208 (1982) 381 ; Z. Phys. C19 (1983) 251;

B.Humpert, Preprint CERN-TH4551 (1986).

[6] P. De Causmaecker, R. Gastmans, W. Troost and T. T. Wu, Phys. Lett. 105B (1981) 215; Nucl. Phys. B206 (1982) 53.

[7] G. Guberina, J. H. Kühn, R. D. Peccei and R. Rück1, Nucl. Phys. B174 (1980) 317.

[8] K. Hagiwara, A. D. Martin and A. W. Peacock, Z. Phys, C (to be published).

[9] E. W. N. Glover, F. Halzen and A. D. Martin, "Physics from $J / \psi-t a g s$ in p $\vec{p}$ collisions", CERN Preprint (to be published).

FIGURE CAPTION

Fig. 1: Feynman diagrams for $g+g \rightarrow{ }^{2 S+1} L_{J}+g$. 

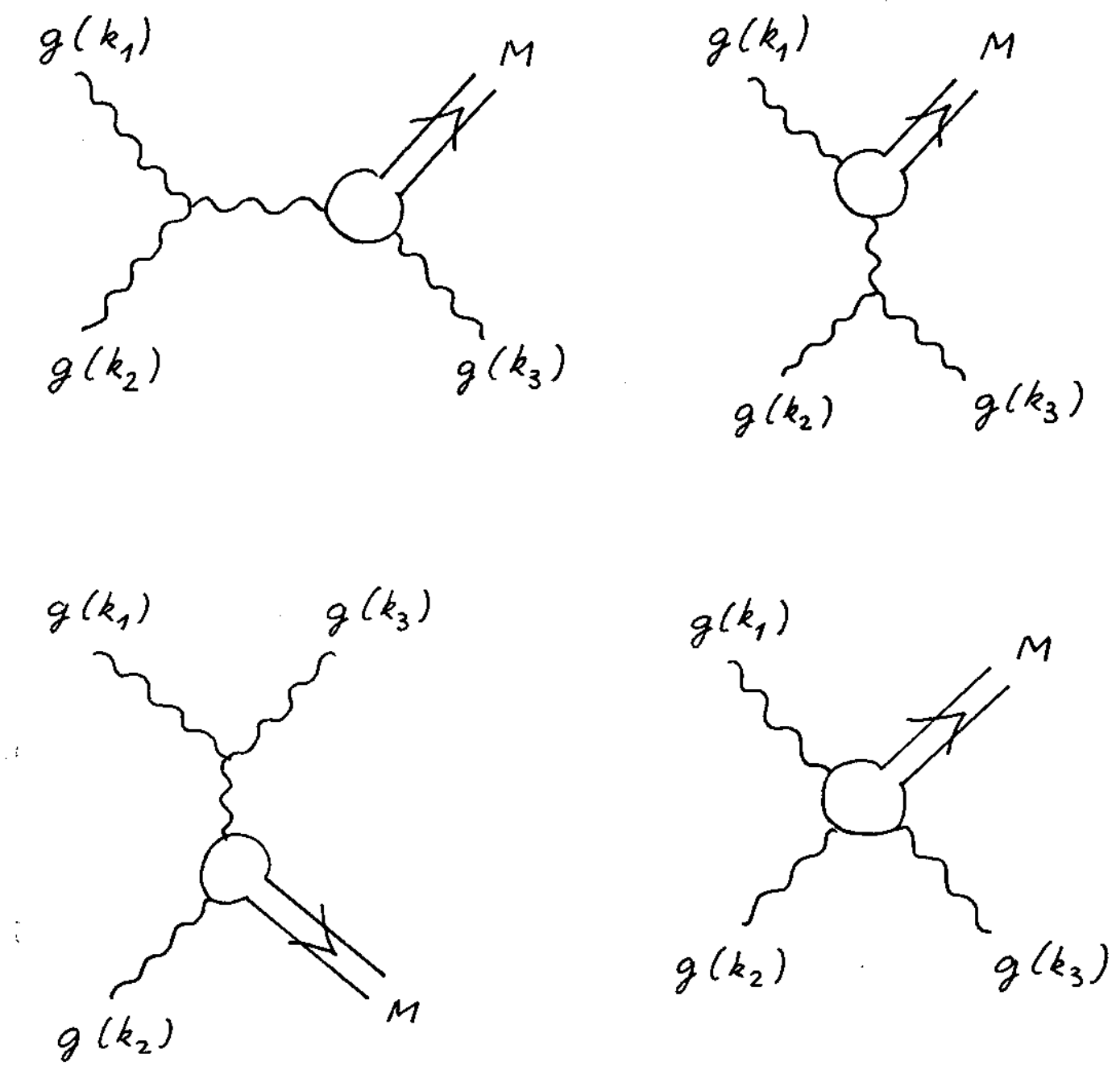

+ permutations 